\title{
Prácticas Eficaces de Liderazgo Dirigidas a Apoyar la Calidad Docente en los CEIP Públicos de Andalucía. La Percepción de Directivos y Maestros
}

\author{
Successful Practices of Leadership Aimed to Support the \\ Quality of Teaching at Andalusian Public Schools. Principals' \\ and Teachers' Perceptions
}

\author{
Marina García-Garnica * \\ Universidad de Granada, España
}

\begin{abstract}
La investigación educativa internacional ha constatado que el liderazgo pedagógico de la dirección es un elemento clave para la mejora de la calidad de las escuelas. Partiendo de esta evidencia, se presenta una investigación que tiene como objetivo conocer las prácticas eficaces de liderazgo educativo que desarrolla la dirección escolar en los CEIPs públicos andaluces, respecto al apoyo a la calidad docente. Para alcanzar este objetivo se ha optado por una metodología cuantitativa, que emplea como procedimiento la encuesta y un cuestionario. La población de esta investigación son los docentes y los equipos directivos de los centros de Educación Infantil y Primaria públicos de Andalucía. Los resultados obtenidos permiten afirmar que la dirección de los CEIPs públicos andaluces desarrolla en un grado elevado las diferentes prácticas dirigidas a apoyar la calidad del trabajo de los docentes, a excepción de aquellas relacionadas con labores de supervisión a nivel de aula, de revisión de programaciones y de concesión de incentivos por un trabajo eficaz. Sin embargo, esta población considera relevante y deseable que este conjunto de prácticas se lleve a cabo. Comparando la opinión de las dos poblaciones participantes en la investigación, puede concluirse que la percepción de lo que ocurre en la escuela con respecto a estas prácticas de liderazgo y la percepción de deseabilidad es significativamente superior en los miembros de equipos directivos en el contexto de la investigación.
\end{abstract}

Descriptores: Liderazgo; Dirección de proyecto; Escuela; Calidad de la educación; Docente.

The international educational research has confirmed that instructional leadership is a key element to improve the quality at school. Starting from this evidence, a research has been carried out to find out successful practices of instructional leadership developed by management teams in Andalusian public schools in regards to the support to teaching quality. To reach this aim a quantitative methodology has been used with the survey as a procedure and a questionnaire. The population of this research are teachers and management teams who work in Ansalusian public schools. The results obtained let us confirm that management teams develop these practices in a high degree, except those related to supervision duties, revision of schedules and granting incentives for effective work. However, this population considers that carrying out this set of practices would be very important and desirable. Comparing the opinion of the two populations participating in the research, it can be concluded that the perception of what happens in the school in regards to the leadership practices and the perception of desirability is significantly higher for the members of management teams.

Keywords: Leadership; Project management; Schools; Educational quality; Teachers.

*Contacto: mgarnica@ugr.es

ISSN: 1696-4713

www.rinace.net/reice/

revistas.uam.es/reice
Recibido: $\quad 11$ de enero 2018

$1^{\text {a }}$ Evaluación: 23 de febrero 2018

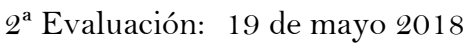

Aceptado: 16 de junio 2018 


\section{Revisión de la literatura}

El liderazgo pedagógico o instruccional es uno de los factores clave en el éxito y eficacia de las escuelas. Este no es un concepto nuevo, sino que surge en la década de los setenta y ochenta del pasado siglo en Estados Unidos, poniendo el foco en la faceta educativa de la dirección.

Consiste, por tanto, en centrarse en los aspectos de carácter pedagógico, dirigiendo la atención hacia todas aquellas acciones que influyen en la labor del profesorado y que afectan al aprendizaje de los alumnos; defendiendo la idea de que mejorar la calidad de los procesos de enseñanza-aprendizaje debe ser la labor prioritaria de la dirección, relegando a un segundo plano la vertiente más técnica y administrativa (Coronel, 1996; González González, 2011; López-Yáñez et al., 2003). Como dice Álvarez (1998), el proceso educativo debe ser el núcleo del liderazgo en un centro que trabaja por la calidad.

Como ya se ha enfatizado (García-Garnica, 2016a), esta tendencia a convertir lo pedagógico en el centro de la labor directiva supone un importante acierto; pues las escuelas, como cualquier otra organización, deben focalizar sus esfuerzos en reconducir la actividad hacia el logro de sus objetivos. En el caso que nos ocupa, las organizaciones educativas, la meta no puede ser otra que optimizar los procesos de enseñanza y garantizar aprendizajes de calidad, y la dirección debe orientar parte importante de su labor a facilitar la consecución de esta meta.

Tampoco es una novedad que el liderazgo es un elemento crucial en la mejora de la calidad de los centros educativos (Seashore-Louis, 2017). Hace ya algunas décadas que la investigación señaló el ejercicio de un buen liderazgo como un rasgo fundamental de las escuelas eficaces. Esta idea, como afirma Villa (2015), se ha ido reforzando durante los siguientes años con múltiples investigaciones en el panorama nacional $\mathrm{y}$, sobre todo, internacional, que justifican y evidencian que el liderazgo pedagógico de la dirección se encuentra entre los factores más potentes con los que cuentan las escuelas para lograr calidad y eficacia (Leithwood et al., 2006).

Por tanto, el liderazgo marca la diferencia (Bolívar, 2012), pues, de los factores escolares que influyen en el aprendizaje de los alumnos, es el segundo en relevancia, estando solo por detrás del trabajo que desarrollan los docentes dentro del aula y llegando a explicar hasta un $25 \%$ de la variación en el aprendizaje que se atribuye a factores educativos (Darling-Hammond, 2012; Leithwood y Seashore-Louis, 2012; Robinson, 2007).

Esto se debe a que los líderes escolares, al moldear y mejorar las condiciones en las que se producen los procesos de enseñanza, tienen la capacidad para influir de forma mediada sobre los aprendizajes de los alumnos (Bolivar, 2010; Hallinger y Heck, 1998; Leithwood, Begley y Cousins, 1990; Leithwood y Seashore-Louis, 2012). Por tanto, como afirman Day y otros (2010), los directores que ejercen el liderazgo pedagógico tienen un efecto directo sobre los docentes, concretamente en la forma en que piensan, programan y efectúan su propia práctica, así como en su nivel de bienestar, confianza y compromiso. En este sentido, la labor de los líderes debe estar dirigida a favorecer que los docentes mejoren su trabajo, influyendo en aspectos como son su capacidad (habilidades y conocimientos), su motivación y compromiso, y las condiciones en las que desarrollan su práctica (Leithwood et al., 2006). 
Siguiendo esta línea argumental, el desempeño de un liderazgo pedagógico eficaz, que permita indirectamente un incremento de la calidad de los aprendizajes, requerirá la puesta en marcha de todo un conjunto de prácticas que permitan influir sobre todos estos aspectos que mejoran la labor de los docentes.

Desde hace algunas décadas, se han desarrollado numerosas investigaciones (Álvarez, 1998; Cawelti, 1987; Day et al., 2010; Day y Sammons, 2013; Leithwood et al., 2006; Pont, Nusche y Moorman, 2009; Stoll y Temperley, 2011) que han tratado de identificar cuáles son estas prácticas eficaces de liderazgo pedagógico que mejoran la calidad de los aprendizajes y marcan la diferencia entre las escuelas. Tras su análisis en profundidad (García-Garnica y Caballero, 2015), se puede afirmar que en todas ellas se hace referencia al mismo tipo de actuaciones, aunque organizadas de distinto modo, en cada caso.

Siguiendo la clasificación establecida por la OCDE (Pont, Nusche y Moorman, 2009; Stoll y Temperley, 2011), quizás una de las más esclarecedoras, es posible identificar cuatro ámbitos esenciales de liderazgo, que integran todo un conjunto de prácticas eficaces: 1. Apoyar, evaluar y fomentar la calidad docente; 2. Establecer metas, evaluar y rendir cuentas; 3. Administrar de forma estratégica los recursos; y 4. Liderar fuera de los límites de la escuela.

La investigación que se presenta está centrada en el primero de estos bloques de prácticas exitosas, que hace referencia a la capacidad de la dirección para centrarse en mejorar la motivación, las capacidades y el contexto en el que trabajan los docentes, a través de acciones que incluyen: la gestión del currículum y las programaciones, la supervisión y evaluación del profesorado, el fomento del desarrollo profesional docente y la creación de una cultura de trabajo colaborativa. En este sentido, el objetivo de este trabajo es conocer las prácticas eficaces de liderazgo educativo que desarrolla la dirección escolar en los CEIPs públicos andaluces para apoyar la calidad de la labor de los docentes.

\section{Método}

Para alcanzar este objetivo se ha optado por una metodología cuantitativa, empleando como procedimiento de investigación la encuesta (Bernardo y Calderero, 2000; Cohen y Manion, 2002), aprovechando su utilidad y eficacia para la descripción y predicción de fenómenos en la investigación educativa (Bisquerra, 2004), así como las numerosas posibilidades y ventajas que ofrece al investigador (Cea D'Ancona, 2001).

La población de esta investigación son los docentes y los equipos directivos de los centros de Educación Infantil y Primaria públicos de Andalucía. La muestra participante y productora de información está compuesta por 533 maestros y 329 directivos escolares, que desempeñan su labor en más de 200 CEIPs andaluces (cuadro 1). Este tamaño es representativo de la población, según la fórmula propuesta por Tagliacarne (1968) considerando una población finita, y su elección ha sido aleatoria, sin responder a un muestreo de conveniencia, garantizando así la validez externa de la investigación (Cea D’Ancona, 2004).

En el caso del colectivo de maestros, la muestra está compuesta en su mayoría por mujeres (77\%), y presenta edades comprendidas entre "menos de 30" y "60 años o más". Los intervalos de edad centrales ("de 30 a 39 años", "de 40 a 49 años" y "de 50 a 59 años") son los más frecuentes, representando un $88 \%$ del total; por su parte, los intervalos situados 
en los extremos ("menos de 30" y “60 años o más”) son los que cuentan con menor representatividad: un $11 \%$ y un $1 \%$, respectivamente ${ }^{1}$.

Cuadro 1. Tamaño de la población y la muestra del estudio

\begin{tabular}{lccc}
\hline & $\begin{array}{c}\text { TAMAÑo DE } \\
\text { LA POBLACIÓN }\end{array}$ & $\begin{array}{c}\text { MUESTRA DE ACUERDo } \\
\text { CON TAGLIACARNE (1968) }\end{array}$ & $\begin{array}{c}\text { MUESTRA } \\
\text { PRODUCTORA DE } \\
\text { INFORMACIÓN }\end{array}$ \\
\hline Maestros & 50.796 & 268 & 533 \\
Equipo directivo & 5.163 & 256 & 329 \\
\hline Fuente: & & & \\
\hline
\end{tabular}

Fuente: Elaboración propia.

En lo relativo a la experiencia como docente, la distribución entre los intervalos establecidos es bastante amplia. Así, el $27 \%$ presenta “de 21 a 30 años de experiencia”, el $23 \%$ "de 11 a 20 años", el $22 \%$ de 5 a 10 años, el 16\% "menos de 5 años" y el 12\% "más de 30 años”. Además, el $31 \%$ afirma tener experiencia en la dirección, habiendo ocupado algún cargo directivo a lo largo de su carrera profesional.

En cuanto a la formación, un $8 \%$ de los maestros encuestados ha realizado otra diplomatura, un 23\% ha cursado una licenciatura, y solo un $4 \%$ ha realizado estudios de posgrado (máster o doctorado). De otra parte, para la población de directivos escolares, se encuentra que de los 329 sujetos que componen la muestra de la investigación el 51\% son mujeres y el $49 \%$ hombres. En relación a la edad, los intervalos centrales (“de 30 a 39 años”, “de 40 a 49 años” y “50 a 59 años”) son los más frecuentes, representando un 97\% del total; mientras que los situados en los extremos (“menos de 30 años” y “60 años o más”) tienen una frecuencia muy reducida.

En lo relativo al cargo, hay que resaltar que el 45\% son directores, el $30 \%$ jefes de estudio y el $25 \%$ secretarios. Y en lo que concierne a la experiencia en el cargo, el 59\% de los encuestados presenta una experiencia superior a los 4 años; a lo que hay que añadir que el $56 \%$ afirma haber ocupado otros cargos directivos con anterioridad. La experiencia docente de los participantes también es amplia: el 64\% lleva más de 20 años dedicado a la docencia, y solo el 1\% tiene menos de 5 años de experiencia.

En cuanto a su formación, un 9\% cuenta con una segunda diplomatura, un 34\% ha realizado una licenciatura y un pequeño porcentaje tiene estudios de posgrado. Además, más de la mitad afirma haber recibido algún tipo de formación específica para desempeñar el cargo que ocupa. El instrumento empleado para desarrollar la investigación es el cuestionario "Prácticas eficaces del liderazgo pedagógico de la dirección escolar" (GarcíaGarnica, 2016b). Su propósito es recoger la opinión que tienen diferentes profesionales de la educación sobre el grado en que los equipos directivos de sus escuelas desarrollan prácticas de liderazgo exitoso, el nivel de relevancia que le otorgan a las mismas y el apoyo que reciben para su desempeño.

Se compone de 83 ítems, de escala valorativa con cinco opciones de respuesta (1. Nada; 2. Poco; 3. Suficiente; 4. Bastante; 5. Mucho), sometidos a una doble valoración para conocer, de una parte, lo que ocurre en el centro respecto a la dirección pedagógica y, de otra, lo que es deseable que ocurra. Estos ítems están organizados en cinco bloques de contenido,

${ }^{1}$ En toda la descripción de la muestra, puesto que se trata de personas, se han redondeado las cifras para poner porcentajes sin decimales. Por tanto, estos datos son aproximados. 
que se corresponden con cinco dimensiones: 1. Capacidad para desarrollar la dirección pedagógica en el centro; 2. Capacidad para compartir las responsabilidades pedagógicas; 3. Formación en habilidades pedagógicas; 4. Convertir la dirección en una profesión atractiva; y 5. Apoyo a las labores pedagógicas de la dirección (cuadro 2). Estas guardan relación con los cuatro ejes establecidos por la OCDE para implementar el liderazgo (Pont, Nusche y Moorman, 2009).

Cuadro 2. Estructura del cuestionario

\begin{tabular}{|c|c|c|}
\hline DIMENSIONES & SUB-DIMENSIONES & ÍTEMS \\
\hline \multirow{4}{*}{$\begin{array}{l}1^{\circ} \text { Capacidad para desarrollar la } \\
\text { dirección pedagógica en el centro }\end{array}$} & Apoyo a la calidad docente & $1-17$ \\
\hline & Gestión estratégica de recursos & $18-26$ \\
\hline & Colaboración más allá de la escuela & $27-33$ \\
\hline & $\begin{array}{l}\text { Fijación y evaluación de metas } \\
\text { educativas }\end{array}$ & $34-42$ \\
\hline $\begin{array}{l}2^{\circ} \text { Capacidad para compartir las } \\
\text { responsabilidades pedagógicas }\end{array}$ & & $43-48$ \\
\hline $\begin{array}{l}3^{\circ} \text { Formación en habilidades } \\
\text { pedagógicas }\end{array}$ & & $49-58$ \\
\hline $\begin{array}{l}4^{\circ} \text { Convertir la dirección en una } \\
\text { profesión atractiva }\end{array}$ & & $59-71$ \\
\hline $\begin{array}{l}5^{\circ} \text { Apoyo a las labores pedagógicas de la } \\
\text { dirección }\end{array}$ & & $72-83$ \\
\hline
\end{tabular}

Fuente: Elaboración propia.

En relación a los criterios de rigor del instrumento, para conocer la confiabilidad se ha utilizado el coeficiente Alfa de Cronbach. Los resultados arrojan, para el grupo completo (docente y directivos escolares), un elevado grado de fiabilidad en todas las dimensiones, tanto en las puntuaciones de la escala "En mi centro", como en las de la escala "Sería deseable". En todos los casos el coeficiente es superior a 0,8, a excepción de una dimensión donde es superior a 0,7 .

También, se ha calculado el grado de fiabilidad de cada una de las dimensiones para las dos poblaciones del estudio por separado, obteniéndose del mismo modo un elevado índice de consistencia interna. Para el estudio de validez del cuestionario se ha empleado la validez de contenido, concretamente se han realizado dos juicios de expertos² (GarcíaGarnica, 2016b).

Para la administración del cuestionario se han diseñado dos formatos del mismo. En primer lugar, se elaboró un cuestionario en formato papel que, por una parte, fue entregado en mano en numerosas escuelas de la provincia de Málaga y Granada aprovechando las ventajas del contacto directo (Van Dalen y Meyer, 1981) y, por otra, fue enviado por correo postal a múltiples centros de toda Andalucía. A partir de este instrumento, y con el propósito de acceder a toda la población de la investigación, se diseñó también una versión on-line, que se envió al correo institucional de todos los CEIPs y se colgó en diversas plataformas profesionales empleadas por este colectivo, siempre acompañado de una carta de presentación. ${ }^{2}$ Todos los datos relativos al proceso de construcción y validación del cuestionario empleado en la investigación pueden
consultarse de forma detallada en el García-Garnica (2016b). 
Los 207 centros participantes en la investigación son públicos, incluyen las etapas de Educación Infantil y Educación Primaria y están ubicados en la comunidad autónoma andaluza.

El análisis de los datos se ha realizado empleando el paquete estadístico SPSS en su versión 20. Así, en primer lugar, se ha llevado a cabo un análisis exploratorio de los datos, basado en el análisis descriptivo de las variables. Este permite conocer, por una parte, la opinión del profesorado sobre las prácticas de liderazgo que desarrolla la dirección en sus escuelas para apoyar la labor docente (escala "en mi centro") y, por otra, la relevancia que este colectivo le otorga al ejercicio de las mismas (escala "sería deseable"). Además, se ha empleado la prueba no paramétrica de rangos con signo de Wilcoxon para muestras relacionadas, con el propósito de estudiar si existen diferencias significativas en la percepción que tienen los maestros sobre lo que ocurre en su escuela y lo que sería deseable que ocurriera, respecto a estas prácticas de liderazgo pedagógico eficaz.

También, se ha efectuado la prueba no paramétricas U de Mann-Whitney para analizar si existen diferencias significativas en la opinión de las dos poblaciones de la investigación con respecto a las labores de apoyo a la calidad docente. Y en último término, se han utilizado las pruebas no paramétricas U de Mann-Whitney y Kruskall-Wallis, para conocer si existen diferencias significativas en la opinión que tiene el colectivo de maestros en función de las variables socio-demográficas establecidas en la investigación.

\section{Resultados}

En esta publicación, se van a aportar resultados relativos a la primera dimensión del cuestionario, concretamente a una de las sub-dimensiones que engloba: "Apoyo a la calidad docente" (ver cuadro 2). Esta categoría, compuesta por un total de diecisiete ítems, hace referencia a la capacidad de la dirección para apoyar e impulsar el potencial de aprendizaje y desarrollo continuo que tiene la escuela como organización, con labores que incluyen la gestión del currículum y el programa de enseñanza, el apoyo y supervisión de la labor docente, el fomento del desarrollo profesional del profesorado o la creación de una cultura de trabajo colaborativa y comprometida con los aprendizajes de todos los alumnos.

Todos los datos que se exponen a continuación corresponden a la población de docentes. Y tan solo en uno de los análisis, se realiza una comparativa entre la percepción de este colectivo y la de los directivos escolares.

En primer lugar, se van a analizar los resultados descriptivos obtenidos para la escala "en mi centro". Tal y como puede observarse en el cuadro 3 y en la figura 1, los ítems incluidos en esta categoría son valorados en conjunto positivamente por el profesorado. En todos ellos la mayoría de los encuestados considera que la dirección de su escuela desarrolla esa práctica eficaz de apoyo a la labor docente en un grado de "bastante o mucho"; y tan solo en los ítems 3, 6 y 7, relativos a la supervisión del trabajo de los maestros en el aula, a la revisión de las programaciones para que permitan aprendizajes de calidad y a la concesión de incentivos a los docentes que realizan su labor con eficacia, las puntuaciones descienden y están más cercanas al grado "suficiente". 
Cuadro 3. Distribución de porcentajes y frecuencias sobre cómo perciben los maestros la capacidad de la dirección para apoyar la calidad docente en su escuela

\begin{tabular}{|c|c|c|c|c|}
\hline & $\begin{array}{l}\text { PoCO- } \\
\text { NADA }\end{array}$ & Suficiente & $\begin{array}{l}\text { BASTANTE- } \\
\text { MUCHO }\end{array}$ & TotaL \\
\hline \multicolumn{5}{|l|}{ La dirección... } \\
\hline $\begin{array}{l}\text { 1. Crea un ambiente de trabajo que } \\
\text { contribuye a la mejora de los aprendizajes }\end{array}$ & $\begin{array}{c}8,2 \% \\
(43)\end{array}$ & $\begin{array}{c}16,6 \% \\
(87)\end{array}$ & $\begin{array}{l}75,2 \% \\
(400)\end{array}$ & $\begin{array}{l}100 \% \\
(525)\end{array}$ \\
\hline $\begin{array}{l}\text { 2. Fomenta el trabajo en equipo entre los } \\
\text { docentes }\end{array}$ & $\begin{array}{l}8,4 \% \\
(44)\end{array}$ & $\begin{array}{l}18 \% \\
(94)\end{array}$ & $\begin{array}{l}73,5 \% \\
(383)\end{array}$ & $\begin{array}{l}100 \% \\
(521)\end{array}$ \\
\hline $\begin{array}{l}\text { 3. Supervisa la labor de los maestros/as en el } \\
\text { aula }\end{array}$ & $\begin{array}{l}24,2 \% \\
(125)\end{array}$ & $\begin{array}{l}48,8 \% \\
(252)\end{array}$ & $\begin{array}{c}26,9 \% \\
(139)\end{array}$ & $\begin{array}{l}100 \% \\
(516)\end{array}$ \\
\hline $\begin{array}{l}\text { 4. Asesora a los docentes en el ejercicio eficaz } \\
\text { de sus funciones }\end{array}$ & $\begin{array}{c}12,9 \% \\
(66)\end{array}$ & $\begin{array}{l}30,8 \% \\
(157)\end{array}$ & $\begin{array}{l}56,2 \% \\
(286)\end{array}$ & $\begin{array}{l}100 \% \\
(509)\end{array}$ \\
\hline $\begin{array}{l}\text { 5. Emplea instrumentos de evaluación } \\
\text { docente apropiados }\end{array}$ & $\begin{array}{l}11 \% \\
(53)\end{array}$ & $\begin{array}{l}32 \% \\
(155)\end{array}$ & $\begin{array}{l}57 \% \\
(276)\end{array}$ & $\begin{array}{l}100 \% \\
(484)\end{array}$ \\
\hline $\begin{array}{l}\text { 6. Revisa las programaciones de aula para } \\
\text { que éstas permitan alcanzar aprendizajes } \\
\text { de calidad }\end{array}$ & $\begin{array}{l}20,9 \% \\
(106)\end{array}$ & $\begin{array}{c}38,5 \% \\
(195)\end{array}$ & $\begin{array}{l}40,5 \% \\
(205)\end{array}$ & $\begin{array}{l}100 \% \\
(506)\end{array}$ \\
\hline $\begin{array}{l}\text { 7. Incentiva a aquellos docentes que realizan } \\
\text { su labor con eficacia }\end{array}$ & $\begin{array}{c}23,9 \% \\
(111)\end{array}$ & $\begin{array}{c}34,2 \% \\
(173)\end{array}$ & $\begin{array}{l}41,9 \% \\
(212)\end{array}$ & $\begin{array}{l}100 \% \\
(506)\end{array}$ \\
\hline $\begin{array}{l}\text { 8. Está disponible cuando el personal docente } \\
\text { lo necesita para resolver cuestiones } \\
\text { pedagógicas }\end{array}$ & $\begin{array}{l}4,8 \% \\
(25)\end{array}$ & $\begin{array}{c}11,8 \% \\
(62)\end{array}$ & $\begin{array}{l}83,4 \% \\
(438)\end{array}$ & $\begin{array}{l}100 \% \\
(525)\end{array}$ \\
\hline $\begin{array}{l}\text { 9. Se reúne con los maestros/as para conocer } \\
\text { sus necesidades formativas }\end{array}$ & $\begin{array}{c}15,5 \% \\
(81)\end{array}$ & $\begin{array}{l}28,7 \% \\
(150)\end{array}$ & $\begin{array}{l}55,8 \% \\
(292)\end{array}$ & $\begin{array}{l}100 \% \\
(523)\end{array}$ \\
\hline $\begin{array}{l}\text { 10. Promueve una formación docente acorde } \\
\text { a las necesidades detectadas en el centro }\end{array}$ & $\begin{array}{c}13,5 \% \\
(70)\end{array}$ & $\begin{array}{c}29,8 \% \\
(154)\end{array}$ & $\begin{array}{l}56,6 \% \\
(293)\end{array}$ & $\begin{array}{l}100 \% \\
(517)\end{array}$ \\
\hline $\begin{array}{l}\text { 11. Desarrolla actividades formativas } \\
\text { dirigidas al profesorado empleando los } \\
\text { recursos disponibles en el centro }\end{array}$ & $\begin{array}{l}20,1 \% \\
(102)\end{array}$ & $\begin{array}{c}29,3 \% \\
(149)\end{array}$ & $\begin{array}{l}50,7 \% \\
(258)\end{array}$ & $\begin{array}{l}100 \% \\
(509)\end{array}$ \\
\hline $\begin{array}{l}\text { 12. Gestiona el currículo con el Equipo } \\
\text { Técnico de Coordinación Pedagógica }\end{array}$ & $\begin{array}{l}6,4 \% \\
(32)\end{array}$ & $\begin{array}{l}20,4 \% \\
(101)\end{array}$ & $\begin{array}{l}73,2 \% \\
(363)\end{array}$ & $\begin{array}{l}100 \% \\
(496)\end{array}$ \\
\hline $\begin{array}{l}\text { 13. Toma decisiones sobre asuntos } \\
\text { curriculares relevantes (contenido, } \\
\text { secuencia, libros de texto...) }\end{array}$ & $\begin{array}{c}9,3 \% \\
(47)\end{array}$ & $\begin{array}{l}29,5 \% \\
(150)\end{array}$ & $\begin{array}{l}61,3 \% \\
(312)\end{array}$ & $\begin{array}{l}100 \% \\
(509)\end{array}$ \\
\hline $\begin{array}{l}\text { 14. Asegura que el currículo se adapte a las } \\
\text { necesidades del centro }\end{array}$ & $\begin{array}{l}7,2 \% \\
(37)\end{array}$ & $\begin{array}{c}20,9 \% \\
(107)\end{array}$ & $\begin{array}{l}71,9 \% \\
(367)\end{array}$ & $\begin{array}{l}100 \% \\
(511)\end{array}$ \\
\hline $\begin{array}{l}\text { 15. Compromete a los docentes con la } \\
\text { atención a la diversidad del alumnado }\end{array}$ & $\begin{array}{l}8,5 \% \\
(44)\end{array}$ & $\begin{array}{c}17,6 \% \\
(91)\end{array}$ & $\begin{array}{l}73,9 \% \\
(381)\end{array}$ & $\begin{array}{l}100 \% \\
(516)\end{array}$ \\
\hline $\begin{array}{l}\text { 16. Apoya al profesorado en su labor de } \\
\text { atención a la diversidad }\end{array}$ & $\begin{array}{l}8,9 \% \\
(46)\end{array}$ & $\begin{array}{l}21,2 \% \\
(109)\end{array}$ & $\begin{array}{c}69,9 \% \\
(360)\end{array}$ & $\begin{array}{l}100 \% \\
(515)\end{array}$ \\
\hline $\begin{array}{l}\text { 17. Promueve el uso de las TICs en el centro } \\
\text { como apoyo a los procesos de aprendizaje }\end{array}$ & $\begin{array}{c}11,4 \% \\
(59) \\
\end{array}$ & $\begin{array}{c}24,7 \% \\
(127)\end{array}$ & $\begin{array}{c}63,9 \% \\
(329)\end{array}$ & $\begin{array}{l}100 \% \\
(515) \\
\end{array}$ \\
\hline
\end{tabular}

Fuente: Elaboración propia.

Por otra parte, para la escala "sería deseable" (cuadro 4 y figura 2), se encuentra que la práctica totalidad de variables que componen esta subdimensión son consideradas por la gran mayoría de los maestros encuestados como "bastante o muy" deseables. Tan solo en el ítem 3, relativo a la supervisión de la labor docente dentro del aula, una parte importante del colectivo de maestros valora la deseabilidad de esta práctica como "suficiente". 


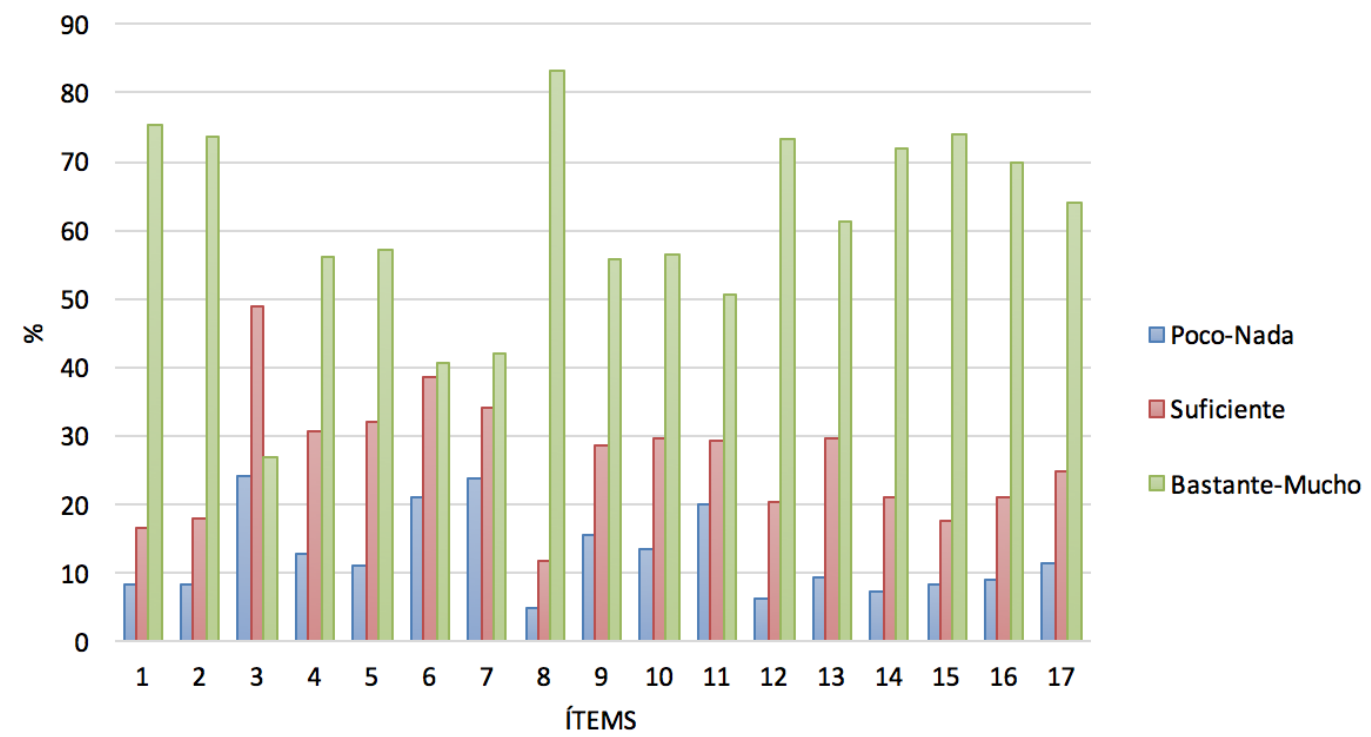

Figura 1. Distribución de porcentajes sobre la opinión de los maestros acerca de la capacidad de la dirección para apoyar la calidad del trabajo docente en su escuela Fuente: Elaboración propia.

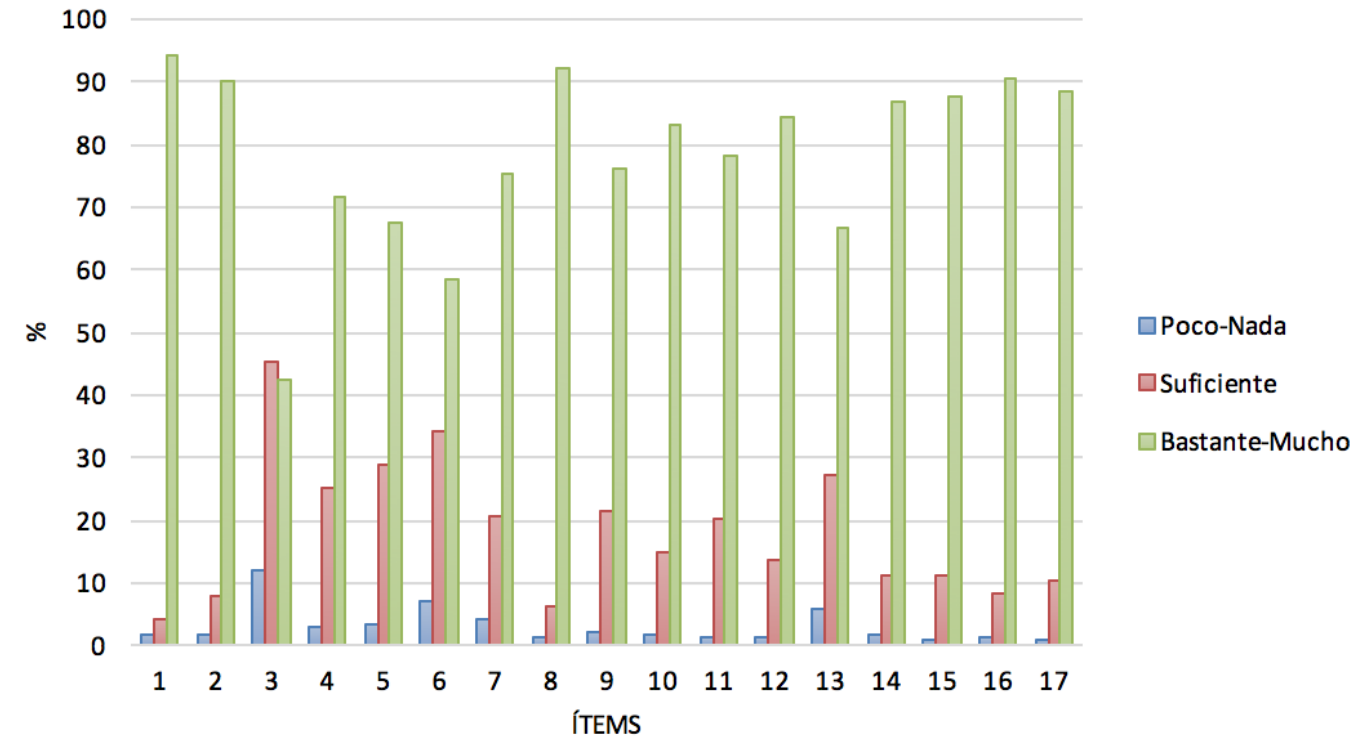

Figura 2. Distribución de porcentajes sobre la opinión de los maestros acerca de la deseabilidad de que la dirección apoye la calidad del trabajo de los docentes en el centro Fuente: Elaboración propia.

Ahora bien, para conocer si existen diferencias significativas en la percepción que tienen los maestros encuestados sobre lo que ocurre en sus escuelas y lo que sería deseable que ocurriera, se ha aplicado la prueba no paramétrica con signo de Wilcoxon para muestras relacionadas. De este modo, se ha podido efectuar una comparación entre ambas escalas para cada uno de los ítems que conforman esta categoría de apoyo a la calidad de la labor docente. 
Cuadro 4. Distribución de porcentajes y frecuencias sobre la importancia que conceden los docentes a que la dirección apoye la calidad de su trabajo

\begin{tabular}{|c|c|c|c|c|}
\hline & $\begin{array}{l}\text { PoCO- } \\
\text { NADA }\end{array}$ & SufiCIENTE & $\begin{array}{l}\text { BASTANTE- } \\
\text { MUCHO }\end{array}$ & TotaL \\
\hline \multicolumn{5}{|l|}{ La dirección... } \\
\hline $\begin{array}{l}\text { 1. Crea un ambiente de trabajo que } \\
\text { contribuye a la mejora de los } \\
\text { aprendizajes }\end{array}$ & $\begin{array}{c}1,6 \% \\
(8)\end{array}$ & $\begin{array}{l}4,2 \% \\
(21)\end{array}$ & $\begin{array}{c}94,2 \% \\
(467)\end{array}$ & $\begin{array}{l}100 \% \\
(496)\end{array}$ \\
\hline $\begin{array}{l}\text { 2. Fomenta el trabajo en equipo entre los } \\
\text { docentes }\end{array}$ & $\begin{array}{c}1,8 \% \\
(9)\end{array}$ & $\begin{array}{l}8 \% \\
(40)\end{array}$ & $\begin{array}{c}90,1 \% \\
(448)\end{array}$ & $\begin{array}{l}100 \% \\
(497)\end{array}$ \\
\hline $\begin{array}{l}\text { 3. Supervisa la labor de los maestros/as en } \\
\text { el aula }\end{array}$ & $\begin{array}{c}12,1 \% \\
(58)\end{array}$ & $\begin{array}{l}45,4 \% \\
(219)\end{array}$ & $\begin{array}{l}42,5 \% \\
(205)\end{array}$ & $\begin{array}{l}100 \% \\
(482)\end{array}$ \\
\hline $\begin{array}{l}\text { 4. Asesora a los docentes en el ejercicio } \\
\text { eficaz de sus funciones }\end{array}$ & $\begin{array}{c}3,1 \% \\
(15)\end{array}$ & $\begin{array}{c}25,4 \% \\
(124)\end{array}$ & $\begin{array}{l}71,5 \% \\
(349)\end{array}$ & $\begin{array}{l}100 \% \\
(488)\end{array}$ \\
\hline $\begin{array}{l}\text { 5. Emplea instrumentos de evaluación } \\
\text { docente apropiados }\end{array}$ & $\begin{array}{c}3,5 \% \\
(17)\end{array}$ & $\begin{array}{l}29 \% \\
(137)\end{array}$ & $\begin{array}{l}67,4 \% \\
(319)\end{array}$ & $\begin{array}{l}100 \% \\
(473)\end{array}$ \\
\hline $\begin{array}{l}\text { 6. Revisa las programaciones de aula para } \\
\text { que éstas permitan alcanzar } \\
\text { aprendizajes de calidad }\end{array}$ & $\begin{array}{l}7,2 \% \\
(35)\end{array}$ & $\begin{array}{l}34,4 \% \\
(168)\end{array}$ & $\begin{array}{c}58,4 \% \\
(285)\end{array}$ & $\begin{array}{l}100 \% \\
(488)\end{array}$ \\
\hline $\begin{array}{l}\text { 7. Incentiva a aquellos docentes que } \\
\text { realizan su labor con eficacia }\end{array}$ & $\begin{array}{l}4,2 \% \\
(20)\end{array}$ & $\begin{array}{c}20,5 \% \\
(99)\end{array}$ & $\begin{array}{l}75,4 \% \\
(365)\end{array}$ & $\begin{array}{l}100 \% \\
(483)\end{array}$ \\
\hline $\begin{array}{l}\text { 8. Está disponible cuando el personal } \\
\text { docente lo necesita para resolver } \\
\text { cuestiones pedagógicas }\end{array}$ & $\begin{array}{c}1,2 \% \\
(6)\end{array}$ & $\begin{array}{l}6,5 \% \\
(32)\end{array}$ & $\begin{array}{r}92,2 \% \\
(454)\end{array}$ & $\begin{array}{l}100 \% \\
(492)\end{array}$ \\
\hline $\begin{array}{l}\text { 9. Se reúne con los maestros/as para } \\
\text { conocer sus necesidades formativas }\end{array}$ & $\begin{array}{c}2,4 \% \\
(12)\end{array}$ & $\begin{array}{l}21,6 \% \\
(107)\end{array}$ & $\begin{array}{c}76 \% \\
(376)\end{array}$ & $\begin{array}{l}100 \% \\
(495)\end{array}$ \\
\hline $\begin{array}{l}\text { 10. Promueve una formación docente } \\
\text { acorde a las necesidades detectadas en } \\
\text { el centro }\end{array}$ & $\begin{array}{c}1,8 \% \\
(9)\end{array}$ & $\begin{array}{c}14,8 \% \\
(73)\end{array}$ & $\begin{array}{l}83,3 \% \\
(410)\end{array}$ & $\begin{array}{l}100 \% \\
(492)\end{array}$ \\
\hline $\begin{array}{l}\text { 11. Desarrolla actividades formativas } \\
\text { dirigidas al profesorado empleando los } \\
\text { recursos disponibles en el centro }\end{array}$ & $\begin{array}{c}1,4 \% \\
(7)\end{array}$ & $\begin{array}{c}20,3 \% \\
(99)\end{array}$ & $\begin{array}{l}78,3 \% \\
(382)\end{array}$ & $\begin{array}{l}100 \% \\
(488)\end{array}$ \\
\hline $\begin{array}{l}\text { 12. Gestiona el currículo con el Equipo } \\
\text { Técnico de Coordinación Pedagógica }\end{array}$ & $\begin{array}{c}1,4 \% \\
(7)\end{array}$ & $\begin{array}{c}13,9 \% \\
(67)\end{array}$ & $\begin{array}{c}84,6 \% \\
(409)\end{array}$ & $\begin{array}{l}100 \% \\
(483)\end{array}$ \\
\hline $\begin{array}{l}\text { 13. Toma decisiones sobre asuntos } \\
\text { curriculares relevantes (contenido, } \\
\text { secuencia, libros de texto...) }\end{array}$ & $\begin{array}{c}5,9 \% \\
(29)\end{array}$ & $\begin{array}{l}27,3 \% \\
(135)\end{array}$ & $\begin{array}{c}66,8 \% \\
(330)\end{array}$ & $\begin{array}{l}100 \% \\
(494)\end{array}$ \\
\hline $\begin{array}{l}\text { 14. Asegura que el currículo se adapte a } \\
\text { las necesidades del centro }\end{array}$ & $\begin{array}{c}1,6 \% \\
(8)\end{array}$ & $\begin{array}{c}11,4 \% \\
(56)\end{array}$ & $\begin{array}{l}87 \% \\
(428)\end{array}$ & $\begin{array}{l}100 \% \\
(492)\end{array}$ \\
\hline $\begin{array}{l}\text { 15. Compromete a los docentes con la } \\
\text { atención a la diversidad del alumnado }\end{array}$ & $\begin{array}{l}1 \% \\
(5)\end{array}$ & $\begin{array}{c}11,2 \% \\
(55)\end{array}$ & $\begin{array}{l}87,8 \% \\
(431)\end{array}$ & $\begin{array}{l}100 \% \\
(491)\end{array}$ \\
\hline $\begin{array}{l}\text { 16. Apoya al profesorado en su labor de } \\
\text { atención a la diversidad }\end{array}$ & $\begin{array}{c}1,2 \% \\
(6)\end{array}$ & $\begin{array}{l}8,2 \% \\
(41)\end{array}$ & $\begin{array}{c}90,5 \% \\
(450)\end{array}$ & $\begin{array}{l}100 \% \\
(497)\end{array}$ \\
\hline $\begin{array}{l}\text { 17. Promueve el uso de las TICs en el } \\
\text { centro como apoyo a los procesos de } \\
\text { aprendizaje }\end{array}$ & $\begin{array}{l}1 \% \\
(5)\end{array}$ & $\begin{array}{l}10,3 \% \\
(51)\end{array}$ & $\begin{array}{c}88,7 \% \\
(438)\end{array}$ & $\begin{array}{l}100 \% \\
(494) \\
\end{array}$ \\
\hline
\end{tabular}

Fuente: Elaboración propia.

Tal y como puede observarse en el cuadro 5, se hayan diferencias significativas en todos los ítems de la subdimensión analizada, siendo la puntuación de deseabilidad superior a la de lo que ocurre en el centro en todos los casos. Llegado este punto, resulta relevante conocer si la percepción que tienen los docentes difiere significativamente de la que tiene la población de directivos escolares en la categoría analizada. Para llevar a cabo este 
análisis, se ha optado por el empleo de la prueba no paramétrica U de Mann-Whitney, pues la distribución de la muestra no sigue una curvatura normal.

Cuadro 5. Contraste de diferencias en la opinión del profesorado entre las escalas en mi centro y sería deseable para el bloque de Apoyo a la calidad docente (Wilcoxon)

\begin{tabular}{|c|c|c|c|c|c|}
\hline & \multicolumn{3}{|c|}{ MAESTROS } & \multicolumn{2}{|c|}{ WILCOXON } \\
\hline & $\mathbf{N}$ & MEDia & DT & $\mathbf{z}$ & $p$ \\
\hline \multicolumn{6}{|l|}{ La dirección.. } \\
\hline $\begin{array}{l}\text { 1. Crea un ambiente de trabajo que contribuye a } \\
\text { la mejora de los aprendizajes }\end{array}$ & 490 & $-0,59$ & 0,99 & $-11,541$ & $0,000^{*}$ \\
\hline $\begin{array}{l}\text { 2. Fomenta el trabajo en equipo entre los } \\
\text { docentes }\end{array}$ & 490 & $-0,53$ & 1,05 & $-10,345$ & $0,000^{*}$ \\
\hline $\begin{array}{l}\text { 3. Supervisa la labor de los maestros/as en el } \\
\text { aula }\end{array}$ & 475 & $-0,42$ & 0,92 & $-9,272$ & $0,000^{*}$ \\
\hline $\begin{array}{l}\text { 4. Asesora a los docentes en el ejercicio eficaz de } \\
\text { sus funciones }\end{array}$ & 472 & $-0,44$ & 1,00 & $-8,725$ & $0,000^{*}$ \\
\hline $\begin{array}{l}\text { 5. Emplea instrumentos de evaluación docente } \\
\text { apropiados }\end{array}$ & 453 & $-0,27$ & 0,89 & $-6,574$ & $0,000^{*}$ \\
\hline $\begin{array}{l}\text { 6. Revisa las programaciones de aula para que } \\
\text { estas permitan alcanzar aprendizajes de calidad }\end{array}$ & 479 & $-0,43$ & 0,95 & $-9,125$ & $0,000^{*}$ \\
\hline $\begin{array}{l}\text { 7. Incentiva a aquellos docentes que realizan su } \\
\text { labor con eficacia }\end{array}$ & 477 & $-0,82$ & 1,25 & $-12,272$ & $0,000^{*}$ \\
\hline $\begin{array}{l}\text { 8. Está disponible cuando el personal docente lo } \\
\text { necesita para resolver cuestiones pedagógicas }\end{array}$ & 490 & $-0,25$ & 0,95 & $-5,440$ & $0,000^{*}$ \\
\hline $\begin{array}{l}\text { 9. Se reúne con los maestros/as para conocer sus } \\
\text { necesidades formativas }\end{array}$ & 492 & $-0,51$ & 1,06 & $-9,701$ & $0,000^{*}$ \\
\hline $\begin{array}{l}\text { 10. Promueve una formación docente acorde a las } \\
\text { necesidades detectadas en el centro }\end{array}$ & 485 & $-0,60$ & 1,00 & $-11,538$ & $0,000^{*}$ \\
\hline $\begin{array}{l}\text { 11. Desarrolla actividades formativas dirigidas al } \\
\text { profesorado empleando los recursos } \\
\text { disponibles en el centro }\end{array}$ & 481 & $-0,65$ & 1,15 & $-11,150$ & $0,000^{*}$ \\
\hline $\begin{array}{l}\text { 12. Gestiona el currículo con el Equipo Técnico } \\
\text { de Coordinación Pedagógica }\end{array}$ & 469 & $-0,24$ & 0,90 & $-5,958$ & $0,000^{*}$ \\
\hline $\begin{array}{l}\text { 13. Toma decisiones sobre asuntos curriculares } \\
\text { relevantes (contenido, secuencia, libros de } \\
\text { texto...) }\end{array}$ & 482 & $-0,16$ & 0,83 & $-4,246$ & $0,000^{*}$ \\
\hline $\begin{array}{l}\text { 14. Asegura que el currículo se adapte a las } \\
\text { necesidades del centro }\end{array}$ & 481 & $-0,37$ & 0,95 & $-8,162$ & $0,000^{*}$ \\
\hline $\begin{array}{l}\text { 15. Compromete a los docentes con la atención a } \\
\text { la diversidad del alumnado }\end{array}$ & 484 & $-0,41$ & 1,02 & $-8,531$ & $0,000^{*}$ \\
\hline $\begin{array}{l}\text { 16. Apoya al profesorado en su labor de atención } \\
\text { a la diversidad }\end{array}$ & 489 & $-0,51$ & 1,07 & $-9,813$ & $0,000^{*}$ \\
\hline $\begin{array}{l}\text { 17. Promueve el uso de las TICs en el centro } \\
\text { como apoyo a los procesos de aprendizaje }\end{array}$ & 488 & $-0,53$ & 1,08 & $-9,993$ & $0,000^{*}$ \\
\hline
\end{tabular}

Nota: * Diferencia significativa $(<0,05)$.

Fuente: Elaboración propia.

Si se observa el cuadro 6, existen diferencias significativas en los ítems 1 y 2 , del 7 al 10 y del 15 al 17 en la percepción que tienen las dos poblaciones sobre la capacidad de la dirección para apoyar la calidad docente en su escuela. Los miembros de equipos directivos tienen una mayor percepción, en su conjunto, en las variables señaladas. Estos ítems hacen referencia a su capacidad para crear un ambiente de trabajo que mejore los aprendizajes (ítem 1), fomentar la colaboración entre docentes (ítem 2), incentivar a aquellos que 
realizan con eficacia su labor (ítem 7), estar disponible cuando se le necesita por cuestiones pedagógicas (ítem 8), reunirse con los maestros para conocer sus necesidades formativas (ítem 9), promover una formación acorde a las necesidades detectadas (ítem 10), comprometer a los docentes con la atención a la diversidad (ítem 15), apoyarlos en esta labor (ítem 16) y promover el uso de las TICs para mejorar los procesos de aprendizaje (ítem 17).

Cuadro 6. Contraste de diferencias entre la percepción de las dos poblaciones para el bloque Apoyo a la calidad docente en la escala en mi centro

\begin{tabular}{|c|c|c|c|c|c|c|}
\hline & \multicolumn{2}{|c|}{ EQUIPOS DIRECTIVOS } & \multicolumn{2}{|c|}{ MAESTROS } & \multicolumn{2}{|c|}{ U MANN-WHITNEY } \\
\hline & Media & DT & Media & DT & $\mathbf{z}$ & p \\
\hline Ítem 1 & 4,23 & 0,62 & 3,97 & 0,93 & $-3,376$ & $0,001 *$ \\
\hline Ítem 2 & 4,37 & 2,29 & 3,95 & 0,95 & $-4,086$ & $0,000^{*}$ \\
\hline Ítem 3 & 2,91 & 0,91 & 2,99 & 0,98 & $-1,861$ & 0,063 \\
\hline Ítem 4 & 3,74 & 0,85 & 3,58 & 1,03 & $-1,595$ & 0,111 \\
\hline Ítem 5 & 3,58 & 0,87 & 3,62 & 0,95 & $-0,597$ & 0,551 \\
\hline Ítem 6 & 3,18 & 1,05 & 3,28 & 1,08 & $-1,272$ & 0,204 \\
\hline Ítem 7 & 3,68 & 1,00 & 3,25 & 1,22 & $-5,053$ & $0,000^{*}$ \\
\hline Ítem 8 & 4,55 & 0,59 & 4,31 & 0,89 & $-2,991$ & $0,003 *$ \\
\hline Ítem 9 & 3,90 & 0,95 & 3,59 & 1,12 & $-3,349$ & $0,001 *$ \\
\hline Ítem 10 & 3,91 & 0,86 & 3,63 & 1,04 & $-3,529$ & $0,000^{*}$ \\
\hline Ítem 11 & 3,64 & 0,96 & 3,45 & 1,16 & $-1,896$ & 0,058 \\
\hline Ítem 12 & 4,06 & 0,89 & 3,99 & 0,94 & $-0,864$ & 0,388 \\
\hline Ítem 13 & 3,77 & 0,90 & 3,78 & 0,99 & $-0,470$ & 0,638 \\
\hline Ítem 14 & 3,98 & 0,83 & 3,96 & 0,99 & $-0,456$ & 0,648 \\
\hline Ítem 15 & 4,29 & 0,71 & 4,01 & $1, \mathrm{OO}$ & $-3,243$ & $0,001 *$ \\
\hline Ítem 16 & 4,37 & 0,67 & 3,97 & 1,04 & $-5,053$ & $0,000^{*}$ \\
\hline Ítem 17 & 4,13 & 0,85 & 3,83 & 1,06 & $-3,742$ & $0,000^{*}$ \\
\hline
\end{tabular}

Nota: * Diferencia significativa $(<0,05)$.

Fuente: Elaboración propia.

En la escala "sería deseable" (cuadro 7), se encuentran diferencias significativas entre la opinión de ambas poblaciones en todos los ítems a excepción del 14. Los miembros de equipos directivos tienen una percepción superior a la del profesorado sobre la deseabilidad de apoyar la calidad de la labor docente en el aula. Por último, es pertinente analizar si existen diferencias significativas en la opinión que tiene la población de maestros en base a las variables socio-demográficas establecidas en la investigación: sexo, edad, años de experiencia docente, otros cargos anteriores, formación otra diplomatura, formación licenciatura y formación posgrado.

Para realizar este análisis inferencial, se ha optado por el uso de pruebas no paramétricas, pues la distribución de la muestra no sigue una curvatura normal. Así, se ha empleado, por una parte, la U de Mann-Whitney para las variables con dos muestras independientes, como el sexo, otros cargos anteriores, formación otra diplomatura, formación licenciatura y formación posgrado; y por otra, Kruskall-Wallis para aquellas con más de dos muestras independientes, como la edad y los años de experiencia docente. A continuación, se comentan los resultados hallados para los ítems que configuran esta categoría (cuadro 8). 
Cuadro 7. Contraste de diferencias entre la percepción de las dos poblaciones para el bloque Apoyo a la calidad docente en la escala sería deseable

\begin{tabular}{|c|c|c|c|c|c|c|}
\hline & \multicolumn{2}{|c|}{ EQUIPO DIRECTIVO } & \multicolumn{2}{|c|}{ MAESTROS } & \multicolumn{2}{|c|}{ U MANN-WHITNEY } \\
\hline & Media & DT & Media & DT & $\mathbf{z}$ & p \\
\hline Ítem 1 & 4,71 & 0,49 & 4,55 & 0,66 & $-3,074$ & $0,002^{*}$ \\
\hline Ítem 2 & 4,70 & 0,50 & 4,46 & 0,74 & $-4,337$ & $0,000^{*}$ \\
\hline Ítem 3 & 3,66 & 0,84 & 3,40 & 0,96 & $-3,720$ & $0,000^{*}$ \\
\hline Ítem 4 & 4,14 & 0,80 & 3,99 & 0,84 & $-2,652$ & $0,008 *$ \\
\hline Ítem 5 & 4,16 & 0,74 & 3,89 & 0,85 & $-4,419$ & $0,000^{*}$ \\
\hline Ítem 6 & 3,98 & 0,80 & 3,68 & 0,92 & $-4,430$ & $0,000^{*}$ \\
\hline Ítem 7 & 4,23 & 0,86 & 4,07 & 0,93 & $-2,382$ & $0,017 *$ \\
\hline Ítem 8 & 4,65 & 0,63 & 4,55 & 0,74 & $-2,277$ & $0,023 *$ \\
\hline Ítem 9 & 4,23 & 0,75 & 4,08 & 0,84 & $-2,306$ & $0,021^{*}$ \\
\hline Ítem 10 & 4,36 & 0,66 & 4,20 & 0,78 & $-2,620$ & $0,009^{*}$ \\
\hline Ítem 11 & 4,26 & 0,76 & 4,10 & 0,79 & $-2,811$ & $0,005^{*}$ \\
\hline Ítem 12 & 4,41 & 0,73 & 4,22 & 0,75 & $-3,889$ & $0,000^{*}$ \\
\hline Ítem 13 & 4,08 & 0,85 & 3,90 & 0,93 & $-2,600$ & $0,009^{*}$ \\
\hline Ítem 14 & 4,38 & 0,78 & 4,30 & 0,75 & $-1,873$ & 0,061 \\
\hline Ítem 15 & 4,64 & 0,60 & 4,41 & 0,75 & $-4,445$ & $0,000^{*}$ \\
\hline Ítem 16 & 4,58 & 0,63 & 4,46 & 0,72 & $-1,975$ & $0,048^{*}$ \\
\hline Ítem 17 & 4,56 & 0,62 & 4,35 & 0,76 & $-4,161$ & $0,000^{*}$ \\
\hline
\end{tabular}

Nota: * Diferencia significativa $(<0,05)$.

Fuente: Elaboración propia.

Cuadro 8. Contraste de diferencias en la opinión de los docentes en función de las variables socio-demográficas

\begin{tabular}{|c|c|c|c|c|c|c|c|}
\hline ÍTEM & SEXo & EDAD & $\begin{array}{c}\text { EXPERIENCIA } \\
\text { DOCENTE }\end{array}$ & $\begin{array}{c}\text { OTROS } \\
\text { CARGOS }\end{array}$ & $\begin{array}{l}\text { FORMACIÓN } \\
\text { DIPLOMAT. }\end{array}$ & $\begin{array}{l}\text { FORMACIÓN } \\
\text { LICENCIAT. }\end{array}$ & $\begin{array}{c}\text { FORMACIÓN } \\
\text { POSGRADO }\end{array}$ \\
\hline$n^{\circ} 1$ & $0,046^{*}$ & 0,085 & 0,118 & 0,297 & 0,360 & 0,157 & 0,206 \\
\hline $\mathrm{n}^{\circ} 2$ & $0,049^{*}$ & 0,151 & 0,125 & 0,079 & 0,630 & $0,045^{*}$ & 0,945 \\
\hline$n^{\circ} 3$ & 0,055 & 0,798 & 0,677 & 0,752 & 0,285 & 0,520 & 0,779 \\
\hline$n^{\circ} 4$ & $0,042^{*}$ & 0,378 & 0,919 & 0,812 & 0,751 & 0,389 & 0,594 \\
\hline $\mathrm{n}^{\mathrm{o}} 5$ & 0,205 & 0,060 & $0,022^{*}$ & 0,485 & 0,541 & 0,090 & 0,097 \\
\hline $\mathrm{n}^{\circ} 6$ & 0,499 & 0,390 & 0,312 & 0,468 & 0,840 & 0,590 & 0,076 \\
\hline $\mathrm{n}^{\circ} 7$ & $0,016^{*}$ & 0,403 & 0,574 & 0,054 & 0,414 & 0,356 & 0,618 \\
\hline$n^{\circ} 8$ & 0,087 & 0,971 & 0,348 & 0,285 & 0,594 & 0,217 & 0,295 \\
\hline $\mathrm{n}^{\circ} 9$ & 0,120 & 0,874 & 0,321 & 0,096 & 0,602 & 0,929 & 0,665 \\
\hline$n^{\circ} 10$ & 0,208 & 0,742 & 0,092 & $0,027 *$ & 0,782 & 0,278 & 0,170 \\
\hline $\mathrm{n}^{\circ} 11$ & 0,761 & 0,091 & $0,045^{*}$ & 0,152 & 0,316 & 0,948 & 0,808 \\
\hline $\mathrm{n}^{\circ} 12$ & 0,628 & 0,063 & 0,193 & 0,286 & 0,387 & 0,856 & 0,743 \\
\hline $\mathrm{n}^{\circ} 13$ & 0,585 & 0,171 & 0,909 & 0,209 & $0,044^{*}$ & 0,192 & 0,326 \\
\hline $\mathrm{n}^{\circ} 14$ & 0,636 & 0,639 & 0,868 & 0,331 & 0,950 & 0,858 & 0,777 \\
\hline $\mathrm{n}^{\circ} 15$ & 0,671 & 0,524 & 0,323 & 0,621 & 0,940 & 0,327 & 0,555 \\
\hline $\mathrm{n}^{\circ} 16$ & 0,599 & 0,336 & 0,136 & $0,009^{*}$ & 0,993 & 0,633 & 0,348 \\
\hline$n^{\circ} 17$ & 0,163 & 0,123 & 0,082 & 0,095 & 0,516 & 0,709 & 0,721 \\
\hline
\end{tabular}

Nota: * Diferencia significativa $(<0,05)$.

Fuente: Elaboración propia.

En el ítem 1, que hace referencia a la capacidad de la dirección para instaurar un clima de trabajo en el centro que favorezca los aprendizajes del alumnado, solo se encuentran 
diferencias significativas para la variable "sexo"; siendo los docentes varones quienes valoran más alto la capacidad de la dirección para desempeñar esta práctica de liderazgo.

En el ítem 2, se hallan diferencias en las variables "sexo" y "formación licenciatura"; siendo los maestros varones y los que no han cursado una licenciatura quienes mejor valoran la capacidad de la dirección para favorecer el trabajo de los docentes en equipo.

Para el ítem 4: "La dirección asesora a los docentes en el ejercicio eficaz de sus funciones", se observan diferencias significativas solo para la variable "sexo". De modo que, nuevamente, los hombres son quienes puntúan más alto esta labor pedagógica de la dirección.

En el ítem 5, se hallan discrepancias en la variable relativa a los "años de experiencia docente". Así, los maestros con más de 30 años de experiencia son quienes mejor estiman la capacidad de la dirección para emplear instrumentos de evaluación docente apropiados.

En el ítem 7 "La dirección incentiva a los docentes que realizan su labor con eficacia", de nuevo, se encuentran diferencias significativas en la variable "sexo"; de modo que los varones dedicados a la enseñanza valoran mejor esta labor directiva que sus compañeras mujeres.

En el ítem 10, referente a la capacidad de la dirección para promover en los docentes una formación que se adapte a las necesidades de su escuela, se encuentran diferencias para la variable "otros cargos anteriores". Así, aquellos maestros que han desempeñado cargos directivos con anterioridad son los que mejor puntúan la capacidad de la dirección para desarrollar esta labor.

En el ítem 11, solo se observan diferencias significativas para la variable "años de experiencia docente". Se halla que la valoración del ítem se reduce significativamente a medida que disminuyen los años de experiencia. Así, los maestros con mayor antigüedad en la enseñanza (más de 30 años) son los que mejor percepción tienen de la capacidad de la dirección para desarrollar actividades formativas dirigidas al profesorado empleando los recursos disponibles en la escuela; mientras que, los que cuentan con menos de 5 años experiencia son los que peor puntúan el ítem.

En el ítem 13 pueden apreciarse diferencias significativas para la variable "formación otra diplomatura”. De modo que, los docentes que han realizado una segunda diplomatura son los que mejor estiman la capacidad de la dirección para decidir sobre aspectos curriculares relevantes.

Para el ítem 16, que hace referencia a la capacidad de la dirección para apoyar al profesorado en su labor de atender a la diversidad, se observan diferencias significativas para la variable "otros cargos anteriores", siendo los docentes que han ocupado en el pasado algún cargo quienes valoran mejor esta práctica de liderazgo.

En el resto de los ítems no se hallan diferencias significativas en la opinión de los docentes para ninguna de las variables independientes establecidas en la investigación. 


\section{Discusión y conclusiones}

En primer lugar, considerando la opinión de los maestros, puede afirmarse que la dirección desarrolla distintas prácticas para apoyar la calidad de la labor de los docentes en sus escuelas. Aunque en conjunto estas prácticas reciben una valoración positiva, este colectivo destaca aquellas dirigidas a crear un buen ambiente de trabajo, a fomentar la colaboración, a estar disponible para resolver cuestiones pedagógicas, a gestionar el currículo, a asegurar que este se adapte a las necesidades del centro, a comprometer a los docentes con la atención a la diversidad y a apoyarlos en esta labor. Sin embargo, los profesores encuestados puntúan peor la capacidad de los equipos directivos para supervisar su trabajo a nivel de aula, para revisar las programaciones y para incentivarlos cuando realizan su labor con eficacia.

Concretamente, la supervisión de la labor de los maestros en el aula es la práctica peor valorada. Esta percepción del profesorado queda avalada por los datos publicados en el Informe Talis, donde se especifica que, aunque existen evidencias claras de que la observación directa en el aula contribuye a la mejora del proceso de enseñanza (INEE, 2014a), en España esta labor es poco frecuente; así, únicamente el 30\% de los directores declara llevarla a cabo (INEE, 2014b).

En conjunto, las tres prácticas que reciben peor valoración por parte de este colectivo, no hacen más que apoyar los resultados de otras investigaciones: en esencia, la dirección tiene poca o ninguna capacidad para intervenir en los procesos de enseñanza en el aula, que quedan al arbitrio de cada docente (Bolívar, Caballero y García-Garnica, 2017). Esta situación, que es completamente insostenible y no puede garantizar el éxito educativo, proviene de una cultura individualista heredada (Viñao, 2004) en la que los aprendizajes están sujetos al voluntarismo de cada profesor, que actúa de forma autónoma y a puertas cerradas en su aula, como si de un reino de taifas se tratara (Fernández Enguita, 2007).

Se puede afirmar que el profesorado considera que es bastante o muy deseable que la dirección apoye la calidad de la labor docente. De hecho, el conjunto de prácticas que integran este bloque son muy bien valoradas por este colectivo, que estima que es muy relevante que la dirección cree un buen ambiente de trabajo, fomente la colaboración, los asesore, los evalúe con instrumentos adecuados, revise las programaciones, los incentive cuando hagan su trabajo con eficacia, este disponible para resolver cuestiones pedagógicas, conozca sus necesidades formativas, les ofrezca una formación acorde a las mismas, gestione el currículo con el equipo técnico de coordinación pedagógica, asegure que se adapta a las necesidades del centro, tome decisiones relevantes al respecto, comprometa y apoye al profesorado en la atención a la diversidad y promueva el uso de las TIC's para la mejora de los procesos de enseñanza. Así, solo hay una práctica de liderazgo exitoso que recibe una valoración menos positiva por parte del profesorado, y es aquella que hace referencia a la supervisión de su labor en el aula.

Como es de esperar, en el conjunto de la dimensión la puntuación es mayor en la escala sería deseable. Así, la visión de deseabilidad es superior a lo que ocurre en la escuela en cuanto a la capacidad para llevar a cabo prácticas de liderazgo dirigidas a apoyar la labor docente.

Además, se puede concluir que los directivos escolares tienen mejor percepción de su capacidad para apoyar la calidad del quehacer docente que los propios maestros. Así, valoran por encima de ellos la labor para crear un buen ambiente de trabajo, para fomentar 
la colaboración, para incentivar a quienes realizan con eficacia su trabajo, para estar disponible en cuestiones pedagógicas, para conocer las necesidades formativas del profesorado y promover una formación acorde a las mismas, para comprometerlo en la atención a la diversidad y para impulsar el uso de las TIC's como apoyo a la enseñanza.

Del mismo modo, se observa que los miembros de los equipos directivos tienen una opinión superior a la de los maestros sobre la deseabilidad de apoyar la labor docente a través de diferentes prácticas eficaces.

Estos resultados no son excepcionales, sino que refuerzan lo que se ha observado en estudios similares (Bolívar, Caballero y García-Garnica, 2017; Bolívar, López y Pérez, en prensa). Así, en la primera de ellas se recoge que:

\begin{abstract}
Normalmente, como puede ser comprensible, el equipo directivo suele ser el que mejor valora su propia actuación, el profesorado mantiene una actitud comprensiva o condescendiente (aunque con una valoración algo menos positiva), mientras que la inspección suele ser la más crítica, valorando más insatisfactoriamente la labor de la dirección. (p. 492)
\end{abstract}

En último término, se ha encontrado que algunas variables socio-demográficas resultan determinantes en la percepción que tiene la población docente sobre este conjunto de prácticas de liderazgo pedagógico exitoso. Concretamente, la variable que resulta más significativa es el sexo, seguida de la experiencia docente y el desempeño de cargos directivos. De modo que los varones, los maestros con mayor antigüedad en la docencia y aquellos que han desempeñado en el pasado labores directivas son quienes mejor valoran la capacidad de la dirección para desempeñar prácticas dirigidas a apoyar la labor de los docentes.

Como se ha venido comentando a lo largo de este trabajo de investigación, el ejercicio del liderazgo pedagógico por parte de la dirección escolar importa y marca la diferencia en las escuelas (Bolívar, 2012). Su impacto en la mejora de los procesos de enseñanza-aprendizaje se ha evidenciado en múltiples investigaciones (Day et al., 2009; Hargreaves y Goodson, 2006; Leithwood, 2009; Maureira, 2004), al tiempo que, como dice Villa (2015), su relevancia queda patente en los innumerable artículos y publicaciones científicas realizadas al respecto. Los resultados expuestos en este trabajo apuntan a que los docentes y especialmente los directivos escolares también son muy conscientes de la importancia que tiene el liderazgo pedagógico y el desempeño de prácticas eficaces asociadas, en este caso, al apoyo de la calidad de la labor del profesorado.

El trabajo que se presenta forma parte de una investigación más amplia de tesis doctoral. Una de las mayores fortalezas de esta investigación es el trabajo de triangulación. Así, se ha recogido la opinión de diferentes profesionales de la educación que trabajan en el contexto estudiado: por una parte, los docentes y, por otra, los miembros de la dirección (director, jefe de estudios y secretario). Pero, además, se ha recurrido al uso de diferentes técnicas de recogida de información: el cuestionario y la entrevista, aunque en este trabajo solo se aportan los resultados obtenidos con el cuestionario, es posible consultar los principales hallazgos de las entrevistas para la dimensión de apoyo a la calidad de la labor docente en otra publicacións.

\footnotetext{
${ }^{3}$ García-Garnica, M. (2018). Prácticas de Liderazgo Orientadas a Apoyar la Calidad de la Labor Docente: La Voz de los Directivos Escolares. En F. J. Murillo (Coord.), Avances en democracia y liderazgo distribuido en educación. Actas del II Congreso Internacional sobre Liderazgo y Mejora de la Educación (pp. 389-392). Madrid: RILME.
} 
Quizás, la mayor limitación es que la investigación se reduce a la comunidad autónoma andaluza, por lo que los resultados no son extrapolables al conjunto de los CEIPs españoles, ni tampoco a otras etapas educativas. No obstante, reporta información relevante en lo relativo al ejercicio del liderazgo pedagógico, que nos ayuda a comprender en qué punto se encuentra y qué progresos se están alcanzando al respecto.

\section{Referencias}

Álvarez, M. (1998). El liderazgo de la calidad total. Madrid: Ed. Escuela Española.

Bernardo, J. y Calderero, J. F. (2000). Aprendo a investigar en educación. Madrid: Rialp.

Bisquerra, R. (2004). Metodología de la investigación educativa. Madrid: La Muralla.

Bolívar, A. (2012). Políticas actuales de mejora y liderazgo educativo. Málaga: Aljibe.

Bolívar, A., Caballero, K. y García-Garnica, M. (2017). Evaluación multidimensional del liderazgo pedagógico: Claves para la mejora escolar. Ensaio: Avaliação e Políticas Públicas em Educação, 25(95), 483-506. https://doi.org/10.1590/s0 104-40362017002500780

Bolívar, A., López, M. C. y Pérez, P. (en prensa). Efficacy of the educational leadership in the spanish context. The perspective of its agents. NASSP Bulletin.

Cawelti, G. (1987, febrero). How effective instructional leaders get results. Comunicación presentada en el Annual Meeting of the American Association of School Administrator. Nueva Orleans, LA.

Cea D’Ancona, M. A. (2001). Metodología cuantitativa: Estrategias y técnicas de investigación social. Madrid: Síntesis.

Cea D’Ancona, M. A. (2004). Métodos de encuesta: teoría y práctica, errores y mejora. Madrid: Síntesis.

Cohen, L. y Manion, L. (2002). Métodos de investigación educativa. Madrid: La Muralla.

Coronel, J. M. (1996). La investigación sobre el liderazgo y procesos de cambio en centros educativos. Huelva: Publicaciones de la Universidad de Huelva.

Darling-Hammond, L. (2012). Educar con calidad y equidad. Los dilemas del siglo XXI. Santiago de Chile: Centro de Innovación en Educación de la Fundación Chile.

Day, C., Hopkins, D., Harris, A. y Ahtaridou, E. (2009). The impact of school leadership on pupil outcomes. Nottingham: National College for School Leadership.

Day, C., Sammons, P., Hopkins, D., Harris, A., Leithwood, K., Gu, Q. y Brown, E. (2010). 10 strong claims about effective school leadership. Nottingham: National College for Leadership.

Day, C. y Sammons, P. (2013). Successful leadership: A review of the international literature. Nottingham: CfBT Education trust.

Fernández Enguita, M. (2007). Dirigir, en España, es morir. Resistencias corporativas y estilos de dirección en los centros docentes. Revista de Educación, 344, 511-532.

García-Garnica, M. (2016a). La relevancia del liderazgo pedagógico para la mejora y la calidad de la educación. Revista Internacional de Didáctica y Organización Educativa, 1(2), 4-20.

García-Garnica, M. (2016b). Elaboración y validación de un cuestionario para medir prácticas eficaces de liderazgo pedagógico de la dirección. Profesorado, Revista de Currículum y Formación del profesorado, 20(3), 493-526. 
García-Garnica, M. y Caballero, K. (2015). ¿Qué prácticas eficaces desarrollan los directivos andaluces en sus escuelas? Revista Iberoamericana de Evaluación Educativa, 8(2), 129-147.

González González, M. T. (2011). Dirección y liderazgo educativo en los centros escolares. En M. T. González (Coord.), Innovación en el gobierno y la gestión de los centros escolares (pp. 163-183). Madrid: Síntesis.

Hallinger, P. y Heck, R. H. (1998). Exploring the principal's contribution to school effectiveness: 1980-1995. School Effectiveness and School Improvement, 9(2), 157-191. https://doi.org/10.1080/0924345980090203

Hargreaves, A. y Goodson, I. (2006). Educational change over time? The sustainability and nonsustainability of three decades of secondary school change and continuity. Educational Administration Quarterly, 42(1), 3-41. https://doi.org/10.1177/0013161X05277975

INEE. (2014a). Talis 2013: Estudio internacional sobre la enseñanza y el aprendizaje. Informe internacional. Boletín de Educación Educainee, 34, 1-4.

INEE. (2014b). Talis 2013: Estudio internacional sobre la enseñanza y el aprendizaje. Informe español. Boletín de Educación Educainee, 33, 1-4.

Leithwood, K. (2009). Cómo liderar nuestras escuelas: Aportes desde la investigación. Santiago de Chile: Fundación Chile.

Leithwood, K. A., Begley, P. y Cousins, J. B. (1990). The nature, causes and consecuences of principal's practices: An agenda for future research. Journal of Educational Administration, 28(4), 5-31. https://doi.org/10.1108/09578239010001014

Leithwood, K., Day, C., Sammons, P., Harris, A. y Hopkins, D. (2006). Seven strong claims about successful school leadership. Nottingham: DfES/ NCSL.

Leithwood, K. y Seashore-Louis, K. S. (2012). Linking leadership to student learning. San Francisco, CA: Jossey-Bass.

López-Yáñez, J., Sánchez, M., Murillo, P., Lavié, J. M. y Altopiedi, M. (2003). Dirección de centros educativos. Madrid: Síntesis.

Maureira, O. (2004). El liderazgo factor de eficacia escolar, hacia un modelo causal. REICE. Revista Iberoamericana sobre Calidad, Eficacia y Cambio en Educación, 2(1), 8-29.

Pont, B., Nusche, D. y Moorman, H. (2009). Mejorar el liderazgo escolar. Volumen 1: Política y práctica. Ciudad de México: OCDE.

Robinson, V. (2007). School leadership and student outcomes: Identifying what works and why. Winmalee: Australian Council for Educational Leaders.

Seashore-Louis, K. (2017). Liderazgo y aprendizajes: Implicancias para la efectividad de las escuelas. En J. Weinstein y G. Muñoz (Eds.), Mejoramiento y liderazgo en la escuela. Once miradas (pp. 150-181). Santiago de Chile: Ediciones Universidad Diego Portales.

Stoll, L. y Temperley, J. (2011). Mejorar el liderazgo escolar. Herramientas de trabajo. Ciudad de México: OCDE.

Tagliacarne, G. (1968). Técnicas y práctica de las investigaciones de mercado. Barcelona: Ariel.

Van Dalen, B. D. y Meyer, W. J. (1981). Manual de técnica de la investigación educacional. Barcelona: Paidós.

Villa, A. (2015). Importancia e impacto del liderazgo educativo. Revista Padres y Maestros, 361, 811.

Viñao, A. (2004). La dirección escolar: Un análisis genealógico-cultural. Educação, 27(2), 367-415. 


\section{Breve $\mathrm{CV}$ de la autora}

\section{Marina García-Garnica}

Doctora por la Universidad de Granada, profesora del Departamento de Métodos de Investigación y Diagnóstico en Educación, miembro del grupo de investigación Ítaca (Investigación y Transformación de Contextos y Aprendizajes). Ha impartido docencia de grado y posgrado, relacionada con el ámbito de la Orientación y la Acción Tutorial, así como con Didáctica y la Organización Escolar. Su investigación se centra en las líneas de trabajo, habiendo realizado diferentes publicaciones de interés científico, tales como artículos, libros y capítulos de libros, así como contribuciones en eventos científicos de carácter nacional e internacional. También, ha participado en diferentes proyectos de investigación con financiación pública y realizado estancias en centros de reconocido prestigio. ORCID ID: https://orcid.org/0000-0002-7417-0294. Email: mgarnica@ugr.es 